\title{
ANÁLISIS DE ERRORES EN LA PRODUCCIÓN ESCRITA DE LOS APRENDICES POLACOS DE ESPAÑOL: LAS PREPOSICIONES
}

\begin{abstract}
Fernández Jódar Raúl, Análisis de errores en la producción escrita de los aprendices polocos de español: las preposiciones [Error analysis on written production of Polish learners of Spanish: the prepositions]. Studia Romanica Posnaniensia, Adam Mickiewicz University Press, Poznań, vol. XXXIII : 2006, pp. 145-157. ISBN 83-232-1643-6, ISSN 0137-2475.
\end{abstract}

The article analyses the prepositional system of Polish learners of Spanish interlanguage at three different levels. The author's intention is to show that in spite of wide prepositional system the errors in the use of prepositions are few. For this reason its teaching in Spanish as a foreign language classes should not create problems.

\section{INTRODUCCIÓN}

La presente investigación se enmarca en un proyecto más amplio de descripción de los errores interlingüísticos morfosintácticos, léxicos y gráficos de los aprendices polacos de español. Los sujetos eran estudiantes del primer ciclo (tres cursos) de Filologia Hispánica de la Universidad Adam Mickiewicz de Poznań entre los cursos 2000-2001 y 2004-2005 pertenecientes al grupo principiante (este grupo es inicial en español y avanzado en francés). A finales del segundo semestre lectivo de cada curso redactaron un texto sobre un tema propuesto' para lo que

${ }^{1}$ Los temas propuestos en los tres difercntes niveles fueron escogidos con la intención de no crear problemas de ideas. Es decir, eran temas lo sufícientemente amplios como para que el aprendiz tuviera siempre algo que explicar. En todo caso, los aprendices fueron instruidos sobre la idea de que el tema era una mera excusa para realizar una composición. Así, por ejemplo, el tema del primer curso era "Un día que cambió tu vida". En relación a este tema se podia recurrir a las vacaciones, los viajes, el trabajo, los estudios, la amistad, la familia, etc. El tema del segundo curso era "Las últimas o tus mejores vacaciones". Sobre este tema se podía recurrir a los viajes, a la descripción de una ciudad, a las diferencias de carácter entre los habitantes de diferentes paises, a la literatura, etc. Finalmente, al tercer curso se le propuso escribir sobre "La transformación de Polonia en los últimos 25 años". Los temas relacionados con esta última propuesta eran quizás no tan amplios, aparentemente, pero la simple 
dispusieron de 25 minutos. Para evitar variantes participaron dos promociones distintas tres años consecutivos ${ }^{2}$.

Del primer curso disponemos de 23 textos, 26 del segundo y 18 del tercero, sumando 67 textos en total. El número varía debido a ausencias el día de la toma de datos y a que se han descartado estudiantes cuya lengua materna sea otra al polaco $o$ sea el español, así como estudiantes que repetieran curso $^{3}$.

\section{PREPOSICIONES ${ }^{4}$}

El uso de las preposiciones suele plantear dificultades tanto para el aprendiz como para el educador por el aspecto idiosincrásico que presentan en las diferentes lenguas. Además, debemos tener en cuenta que en el caso de los sujetos de la investigación se superponen en la interlengua tanto el sistema preposicional del polaco como del francés. Esta dificultad ${ }^{5}$ se refleja en el alto número de errores (189), aunque también es cierto que existe una elevada frecuencia de uso, como se observa en las figuras 1 y 2 , y que los errores están repartidos en una amplia lista de preposiciones y locuciones preposicionales. En global, los 189 errores representan el $9,064 \%$ del total de errores o el $11,969 \%$ si no tenemos en cuenta los errores gráficos.

Poner en paralelo los errores y la frecuencia de uso nos debería servir para relativizar la importancia real de los errores prepositivos. En la figura 1 observamos como la frecuencia de uso aumenta claramente junto con la producción total de

descripción de las diferentes realidades de la sociedad polaca antes y ahora daba mucho juego a los aprendices. Por lo tanto, la idea principal a la hora de escoger los temas no era tanto que les provocara especial motivación, sino el hecho de que fueran to menos limitadores posibles, pero que al mismo tiempo les obligara a elaborar una mínima narración.

${ }^{2}$ Ya en Fernández Jódar (2004) observamos la importancia del carácter colectivo a la hora de realizar una toma de datos de grupos y como éstos podian falsear los resultados. Cuando un grupo es participativo y comunicativo, la producción será mayor y, en cambio, si es tímido o poco receptivo la producción será menor. En el caso de los grupos participantes en nuestra investigación podriamos definirlos como no problemáticos y participativos.

${ }^{3}$ Siempre es recomendable el mayor número posible de sujetos. Aun así, recordemos que en la reconocida investigación de Fernández (1997) se trabajó con un corpus de 108 textos de aprendices de cuatro L1 diferentes (alemán, árabe, francés y japonés) y de tres niveles distintos, por lo que había una media de 9 textos por nivel y Ll. Nosotros trabajamos únicamente con aprendices cuya Ll es el polaco y la media por nivel sería de 22,33 textos. Es, por tanto, un corpus más amplio.

${ }^{4}$ En este punto tratamos tanto las preposiciones como las locuciones preposicionales. La distinción entre ambas y sus características aparecen descritas en los capítulos pertinentes de las gramáticas referidas en la bibliografia. Por otra parte, el estudio conjunto de ambas parece más lógico y cómodo desde el punto de vista didáctico.

${ }^{5}$ Quizá sea esta dificultad la que ha provocado que sea la preposición menos tratada por la bibliografia. En González Cremona y Palka (2004) encontramos un listado ejemplificativo de errores preposicionales en textos de aprendices polacos de español. 
palabras, mientras desciende el número de errores, ya de por sí considerablemente bajo en comparación al dato anterior.

Figura 1. Número de errores y frecuencia de uso de preposiciones por redacción

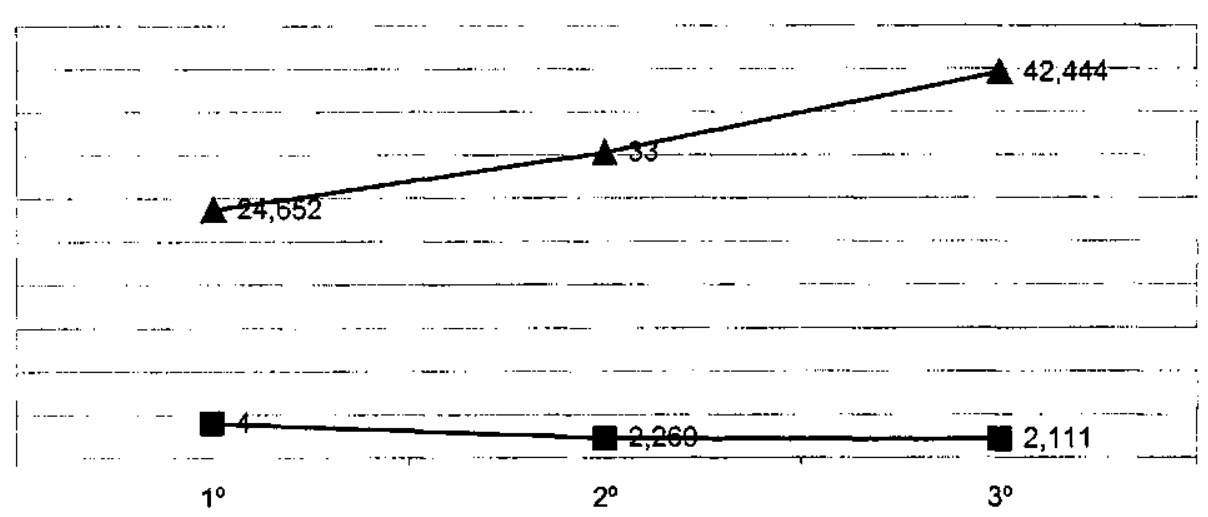

- Errores Arecuencia de uso

En la figura 2 los datos confirman el descenso de errores, mientras que la frecuencia de uso aumenta ligeramente y se estabiliza en el segundo y tercer curso. Recordemos que la longitud analizada en esta figura no varía.

El descenso en el número de errores entre el segundo y el tercer curso en este caso no se estanca pero sí es menor que el descenso entre primero y segundo.

Figura 2. Número de errores y frecuencia de uso de preposiciones por cada 100 palabras

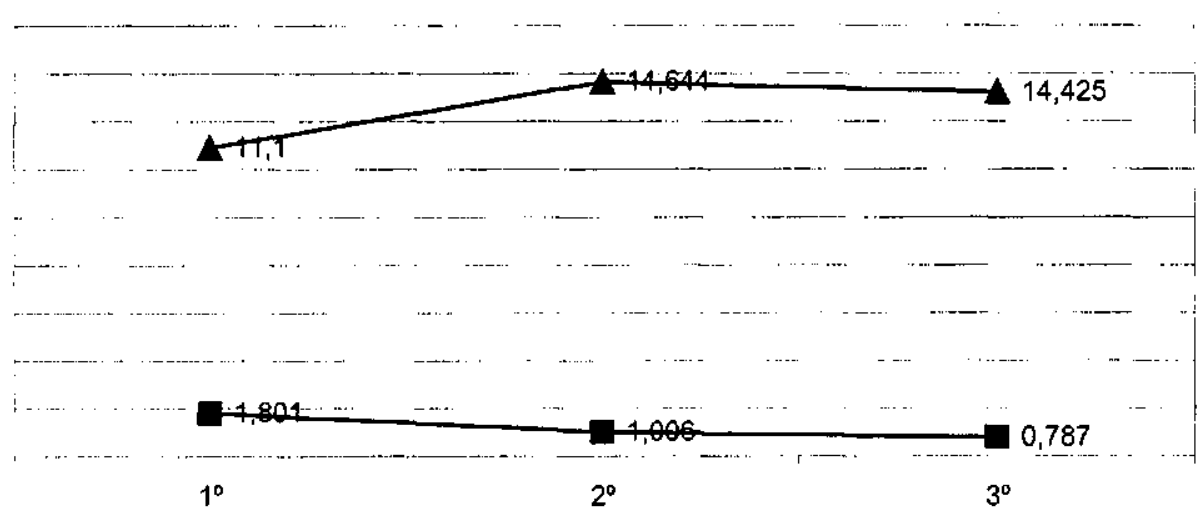

- Errores $\quad \boldsymbol{A}$ Frecuencia de uso 
Las preposiciones y locuciones preposicionales utilizadas suman 2189 repartidas de la siguiente forma:

$$
I^{\circ}
$$

Más de 200 apariciones

Preposición "de"

Preposición "en"

Preposición "a"
Entre 100 y 200 apariciones

$\begin{array}{ll}\text { Preposición "con" } & 46 \\ \text { Preposición "para" } & 38 \\ \text { Preposición "por" } & 22\end{array}$

\section{Entre 5 y 100 apariciones}

Preposición "durante"

Preposición "desde"

Preposición "sin"

Loc. prep. "después de"

Preposición "sobre"

Preposición "hasta"

Preposición "cntre"

Loc. Prep. "cerca de"

Total por cursos

\section{6}

38

22 $2^{\circ}$

286

172

134 $3^{\circ}$

319

139

89

50

47

35

62

786

415

332

Total

76
15
32




$\begin{array}{lrrr}\text { Preposición "para" } & 4 & 139 & 2,877 \% \\ \text { Preposición "por" } & 11 & 119 & 9,243 \% \\ \text { Preposición "desde" } & 2 & 28 & 7,142 \% \\ \text { Loc. Prep. "después de" } & 1 & 19 & 5,263 \% \\ \text { Preposición "sobre" } & 2 & 18 & 11,111 \% \\ \text { Loc. Prep. "junto a" } & 1 & 2 & 50 \% \\ \text { Loc. Prep. "al cabo de" } & 1 & 3 & 33,333 \%\end{array}$

Como observamos, de las preposiciones más frecuentes el mayor porcentaje de error recae sobre "a", seguida de "en", "por", "de", "con" y "para".

\subsection{PREPOSICIÓN "DE"}

La preposición "de" es la que reúne mayor número de errores, debido también a que es la más frecuentemente utilizada. Hay contabilizados 64 errores de los que 18 lo son por elección, 23 por uso innecesario y 23 por omisión. En la tabla inferior observamos como la evolución es decididamente positiva en los tres cursos:

\section{$N^{*}$ de errores $\quad N^{\prime \prime}$ de apariciones Porcentaje de error}

$\begin{array}{llll}\text { Primero } & 29 & 181 & 16,022 \% \\ \text { Segundo } & 24 & 286 & 8,391 \% \\ \text { Tercero } & 11 & 319 & 3,448 \%\end{array}$

- El principal problema es la regencia al sumar 31 errores (14 en primero, 14 en segundo y 3 en tercero). De éstos, 24 son en regencia verbal, 3 de adjetivos y 4 de sustantivos. Los errores se repiten en los siguientes $\operatorname{casos}^{6}$ :

"pensar de" por "pensar en" (cinco veces, aunque en tres textos):

"no pienso de la situación económica en unos países africanos africanos o más bien de la aumentación de los precios" (I.13.), "no pienso mucho del futuro" (I.14.), "Todo el tiempo pensaba de Jorge y de su vida triste en el bar" (1.22.)

"decidir de" por "decidir" (cuatro veces):

"decidió de viajar a Africa" (I.23.), "me decidí de realisar un viaje a España" (II.4.), "mis padres decidieron de partir a Italia" (11.22.), "hoy cuando, quizá, puedamos hacer todo, decidir de nuestro destino" (III.4.)

"empezar de" por "empezar a" (dos veces):

"empecé de sospechar que había venido la mañana" (I.13.), "La mayoría empezaba de trabajar justo después de haber aprobado (o no) el bachillerato" (III.10.)

' Tras cada ejemplo aparece entre paréntesis el curso y el número de texto al que pertenece en el corpus de trabajo. 


\section{Otros ejemplos son:}

"Terminamos _ mirar la exposición" (I.5.), "propusieron a los estudiantes de mi clase de hacer teatro" (I.11.), "todo el año soñaba de dos semanas en Italia" (II.5.), "volvía de vivir" (II.6.), "en general todos intentan de partir" (II.15.)

- Otro problema destacable es la omisión de la preposición "de" precediendo a oraciones subordinadas. Este error se produce en 11 ocasiones repitiéndose dos estructuras. En cuatro ocasiones se repite la estructura "tener + sustantivo + de + oración subordinada":

"Tenia la sensación _ que no soportaría más" (1.6.), "tengo esperanza _- que la España me guste tanto como la Francia" (J.10.), "tuvo impresión _ que me asfixiaba" (1.12.), "no tenía ni idea _ que la cuidad puede tener más colores” (III.7.)

En tres ocasiones se repite "estar seguro de + oración subordinada":

"Estoy seguro _ que ya habéis pensado que extraordinario pasó" (I.13.), "estoy segura _ que antes de la accesión de la Polonia a Unión Europea este sueño realizará" (I.14.), "estaba seguro __ que durante este viaje necesitaría trabajar" (II.14.)

\section{Otros ejemplos:}

"Me enteró _ que era mujer" (I.2.), "con la consciencia _, que la temperatura al exterior ya he pasado $-50^{\circ} \mathrm{C}$ " (II.23.), "todo esto resulta del hecho _ que este país se distingue por el número de las naciones que viven ahi" (II.25.), "Esta Barbie era un signo _ que algo va a cambiar" (III.16.)

- En ocho ocasiones (tres en primero y cinco en segundo) se omite "de" en locuciones preposicionales o se usa innecesariamente junto a otras preposiciones:

"dentro _; el cuerpo"(1.2.), "vivía cerca _ me" (1.8.), "tiene cerca _ 34 años" (1.22.), "Salvo de 4 días" (II.6.), "salvo de mi tío" (I.14.)

- En otras cinco expresiones adverbiales su uso es erróneo, en cuatro por elección y en una por uso innecesario:

"De otra parte" (II.19.), "desde del punto de vista de la niña" (III.7.), "Quería que me cuentes todo de tu perspectiva" (III.11.), "aprovechar de máximo la libertad" (III.13.), "puedo hablar pero del punto de vista de una niña" (III.16.)

- En tres ocasiones aparecen errores en el uso u omisión de la preposición "de" con valor partitivo:

"Tenía un poco _ miedo" (I.4.), "Tenía un poco _ miedo" (1.12.), "cada día trae algo de nuevo" (I.15.)

- Encontramos dos errores en la indicación de las fechas:

“el 19 de junio _ 1998” (I.12.), "el lunes de 24 de abril de 1998" (I.22.) 
- También encontramos dos errores en la elección de la preposición "de" por "a" para indicar lugar:

"la direción de Buenos Aires" (I.22.), "era una vuelta del mundo" (II.23.)

- Otros errores con una sola aparición son la omisión en un nombre propio, el uso por la preposición "por" y la omisión de "de" con valor de especificación temporal:

"pasaré unos dias visitando El Museo Prado" (II.21.),"de lo que me cuentan mis padres y la gente mayor, la vida no era tan rosa" (III.7.), "la realidad en Polonia _ hace 25 años" (III.10.)

\subsection{PREPOSICIÓN "EN"}

La preposición "en" es la tercera en cuanto a número total de errores y la segunda en porcentaje entre las seis más utilizadas. Por otra parte, si observamos la tabla inferior, veremos que si bien entre primero y segundo la evolución es favorable, entre segundo y tercero sufre un ligero retroceso:

\section{$N^{0}$ de errores $\quad N^{0}$ de apariciones Porcentaje de error}

$\begin{array}{lllr}\text { Primero } & 18 & 104 & 17,307 \% \\ \text { Segundo } & 12 & 172 & 6,976 \% \\ \text { Tercero } & 11 & 139 & 7,913 \%\end{array}$

- El principal problema se centra en el régimen preposicional de verbos y sustantivos. Suman 21 errores ( 11 en primero, 5 en segundo y 5 en tercero) de los 41 contabilizados y se desglosan de la siguiente manera:

En 10 ocasiones se usa "en" por "a" en regencia de verbos y sustantivos de movimiento como "viajar", "viaje", "venir", "ir" y "volver":

"cada mes hacabamos un viaje en zoo, en bosque o más lejos a Biskupin" (I.17.), "Cuando venía en Polonia, no sabé vivir aqui" (I.18.), "en junio fui en Alemaña a Bad Oyenhayusen" (II.1.), "En septiembre volví en Polonia" (II.6.), "viajé con mis amigos y mi novio en España" (II.17.)

En 4 casos se usa "en" como "por" en regencia del verbo "pasear" o del sustantivo "paseo":

"Un dia paseaba en un parque" (I.3.), "Solabamos dar un paseo en el parque Solacki" (I.17.), "Paseé en la Wall Street" (II.6.), "un negro o un árabe paseando en la calle evoca sensación" (III.11.)

Los casos restantes afectan a la regencia verbal y se refieren a usos de "en" por "a" y por "de" y a omisión de "en": 
"habian asistido en el anuncio de los resultados" (I.S.), "No es tán facil responder en esta cuestión" (I.15.), "la única cosa en que pensé fue _. la engañadora Concepción" (1.23.), "la luz de democracia penetró el ambiente polaco" (III.2.), "veces no había razones para profundizar demasiado _ la realidad" (III.15.)

- Otra elección errónea generalizable es el uso de "en" por "de" con valor de pertenencia o cualidad por interferencia del polaco. Esto ocurre en 14 ocasiones con igual importancia en los tres cursos ( 6 en primero, 4 en segundo y 4 en tercero):

"aprendía muchísimo para no suspendèr los examens a la univesidad en Poznañ" (1.6.), "el parque Solacki, más romántico en Poznań" (1.17.), "me desperté a las seis de la mañana por la causa del terrible ruido en la calle" (I.19.), "eran las más aburridas vacaciones en mi vida" (II.6.), "Me ocupaba de 2 niños en una familia francesa en el sur del pais" (II.9), "la caida del muro 트 Berlín" (III.2.)

- Encontramos también cuatro casos de errores en expresiones adverbiales:

"me puso en muy mal humor" (I.19.), "participé en el mismo tiempo en las clases de baile hindú" (II.8.), "eso implicaba en ojos de los gobernantes, la traición del sistema" (III.2.), "En los principios de los años '80" (III.16.)

- Finalmente, hay dos casos de uso erróneo de "cn" con valor locativo por "bajo" en II.23., y "por" en II.17., el cual también podría ser entendido como un cruce con "en todas las direcciones":

"al lado de la farol que echaba su luz en todas partes" (II.17.), "el hielo reluciendo en el sol" (II.23.)

\subsection{PREPOSICIÓN "A"}

La preposición "a" es la que presenta un mayor porcentaje de errores según hemos visto. De los 53 errores registrados 31 corresponden a omisión, 16 a elección y 6 a uso innecesario. Por cursos observamos como entre primero y segundo la evolución es positiva mientras que entre segundo y tercero hay un estancamiento:

\section{$\mathbf{N}^{0}$ de errores $\quad \mathbf{N}^{n}$ de apariciones Porcentaje de error}

$\begin{array}{lrrr}\text { Primero } & 30 & 109 & 27,522 \% \\ \text { Segundo } & 14 & 134 & 10,447 \% \\ \text { Tercero } & 9 & 89 & 10,112 \%\end{array}$

- El principal problema que aparece es el uso u omisión de la preposición delante de objetos directo. Se registra este error en 17 (12 en primero, 2 en segundo y 3 en tercero) ocasiones.

De éstas, nueve corresponden a omisión de la preposición delante de objeto directo persona: 
"no quería molestar _ mi hermana" (I.19.), "decidió de viajar a Afríca para encontrar _ mi médico" (I.23.), "Echaba de menos _ mis cercanos" (II.17.), "limitaba en sus derechos los ciudadanos polacos" (III.4.)

En tres se omite delante de nombre colectivo:

"podía viajar mucho y encontrar _ mucha gente extranjero: de Alemania, de Austria, de Inglaterra, de Italia, de Francia y _ mucha más" (I. I I.), "podría ayudarme y _ mi familia" (I.14.)

En dos se omite frente a nombre de cosa personificado:

"Yo llamaba _. Angelito durante una hora sin resultados. Cuando era muy lejos de mi casa yo vi _Angelito" (1.12.)

En cuatro se usa innecesariamente ya sea por no referirse a personas o por referirse a personas no específicas:

"espero todo el dia a sus teléfonos" (I.9.), "a otros parczco a un tipo antipático" (I.13.), "me interesa mucho a la naturaleza, a los animales" (I.18.)

- Otro problema que presenta numerosos casos es el referido a la regencia, principalmente verbal. De los diez errores registrados (seis en primero y cuatro en segundo) nueve lo son por omisión y uno por uso innecesario:

"he empezado _ actuar" (I.11.), "estaba acostumbrado _ jugar" (1.17.), "comenzó __ contar el historia de su vida" (1.22.), "me permitió aprender _ ser responsable" (II.12.), "me permitieron a cerrar del interior las puertas del paraiso" (II.13.), "disfrutabamos del tiempo hablando y jugando ajedrez" (11.23.)

- En nueve ocasiones (cinco en primero y cuatro en segundo) encontramos el uso de "a" por "en" para expresar situación espacial:

"fue mi primer dia à la escuela" (I.4.), "querían trabajar al extranjero" (I.10.), "no estudio mucho a mi casa" (I.16.), "Trabajé al campo" (II.6.), "las últimas vacaciones pasé al extranjero" (II. 10.)

- En cuatro ocasiones se utiliza "a" por "de", para indicar cualidad, materia o especificar el tiempo:

"aprendia muchísimo para no suspender los examens a la univesidad" (I.6.), "entraba a mi cuadro todo a madera un pajarillo" (II.13.), "conocía perfectamente los números de teléfonos a las embajadas" (II.16.), "la buena vida a finales de los años setenta" (III.1.)

- Registramos tres errores relacionados con la indicación de tiempo:

“A las 9 y 20 oh no ¡Otra vez! Un día él va a matarme!” (1.7.), “_E El día siguiente, en Noticias de tele, dijeron que el bosque fue destruido" (II.26.), "_ el día siguiente me tenía que levantar pronto" (III.15.)

- Hay sólo dos que afecten a expresiones adverbiales:

“A mi opinión la primera clase era muy aburrida" (1.4.), "Ahora el tener todo el alcance de la mano, una naranja no nos da tanta alegría" (III.9.) 
- Aparecen cinco errores en los que se confunde dirección, distancia, indicación de movimiento y límite, y se omite la preposición "a" o se usa por otra:

“me fue allí_donde había ido anteayer y _donde, pensaba yo, también iré mañana" (I.13.), "Decidí dar un pasea al centro de la ciudad que está muy cerca de mi casa" (I.19.),

"voy a ligar y comer al fin del mundo" (1.21.) "está sítuado apenas 100 kilometros" (II.1.)

- Finalmente, encontramos un único caso en el que se omite la preposición en la estructura "al + infinitivo" para expresar causalidad:

"Ahora el tener todo el alcance de la mano, una naranja no nos da tanta alegría" (III.9.)

\subsection{PREPOSICIÓN "CON"}

De las nueve apariciones erróneas de la preposición "con", seis corresponden a elección, dos a uso innecesario y una a omisión. Son cifras bajas comparadas con la frecuencia de uso.

- Esta preposición indica medio, instrumento o modo de hacer algo, las circunstancias con que se realiza alguna cosa, o compañia. En cambio, en cuatro ocasiones es utilizada en sustitución de la preposición "de" para indicar cualidad, naturaleza o condición de persona o cosa, por interferencia del polaco:

"bocadillos con zanahoria" (1.21.), "todas las películas con Johny" (1.21.), "solamente nosotros con las almas jóvenes y vivas" (II. 17.), "una familia con seis hijos" (III.8.)

- Las causas de los errores en los otros casos son diferentes. En dos se confunde la función de objeto directo y sujeto por un complemento circunstancial y se utiliza "con" innecesariamente. En otro se omite la preposición al confundir un sustantivo por un adjetivo. En otro se otorga a "con" el valor de oposición correspondiente a "contra". Y, finalmente, en un caso denota precio o cuantía, valor correspondiente a "por":

"Me encantaba con su tactos sensibles" (1.3.), "Quizás, el año que viene ¿tenga posibilidad descubrirlas y compartir con usted con mis sentimientos?" (1I.5.), "el único contacto extranjero" (III.10.), "esforzandome con el viento" (II.3.), "limpiaba la casa del jefe con el mismo sueldo" (II.10.)

\subsection{PREPOSICIÓN "PARA"}

Las preposiciones "para" y "por" suelen ejercitarse conjuntamente por considerarse que son causa de confusiones. En los textos se recogen casos así, pero se centran en el uso de "por" por "para" y no al contrario, lo que sucede en una única ocasión. Además, el bajo número de errores frente a la frecuencia de uso denota poca dificultad en su adquisición. 
De los cuatro usos erróneos de esta preposición uno es por uso innecesario al convertir una oración sustantiva en una final, y los otros tres por elección errónea al considerar el valor final de la preposición por encima de otros aspectos. En un caso se debería utilizar "por" ya que expresa "en lugar de" y en los otros dos los sustantivos rigen una preposición diferente:

"Era suficiente para que estuviera fuerte" (I.3.), "provocó unas situaciones raras, para no decir ridiculas" (II.2.), "sin ningúna gana para nada" (II.5.), "tenía descuentos para los billetes de transporte común" (III.8.)

\subsection{PREPOSICIÓN "POR"}

La preposición "por" sí presenta cierta dificultad en comparación a "para", tanto en el porcentaje de errores como en el número total de éstos. Suman once de los que sólo uno es por omisión en una locución adverbial:

"Me enteró que era mujer, siempre (lo-la) era pero _ dentro" (I.2.)

Los otros diez casos son por elección.

- En cuatro ocasiones se confunde con "para", lo que al contrario sucedía en una única ocasión, como vemos arriba:

"Pienso que esa decisión es quasi imposible por los jovenes" (I.4.), "había mucha promociones por nuevos miembros" (I.9.), "tenía que prepararme por el exámen" (II.2.), "Las transformaciones de los últimos 10 años son, indudablemente, más por mi generación, se trata de la etapa de cambios visibles y fascinantes" (III.5.)

- En tres ocasiones se confunde con "a". Una como preposición indicadora de objeto directo y las otras dos en expresiones adverbiales:

"No se puede buscar por los amigos" (I.S.), "me desperté a las seis de la mañana por la causa del terrible ruido" (I.19.), "El regimén socialista arruinaba el país de manera intrínseca, paso por paso" (III.2.)

- Finalmente, en tres ocasiones se confunde con la preposición "de", ya sirva ésta para determinar el tiempo o sea por regencia:

"lo más importante por toda la vida" (I.4.), "un habitante de Quebec que estaba ocupado por el teatro" (I.11.), "sera en Africa por vacaciones" (I.23.)

\subsection{OTRAS PREPOSICIONES}

Los errores referentes a otras preposiciones son muy poco cuantiosos al sumar sólo siete casos. Seis de ellos son errores por elección y el otro por uso innecesario. Son debidos a diferentes causas no generalizables. Aparecen errores de tipo léxico al confundirse "junto a" por "entre", "al cabo de" por "después de" o "después de" por "antes de". En otro se usa "sobre" por "de" restando el valor cualificador de la 
preposición. En dos ocasiones el error se centra en "desde". En una por uso innecesario junto a "hace" $\mathrm{y}$ en otro por combinatoria errónea con "a":

"estaba loca sobre los hombres" (I.3.), "vi un annuncio sobre trabajo (I.10.), han creido un grupo de teatro francés junto a esta escuela y una sección de lenguaje francés" (1.11.), "시 cabo del año estudiantil (...) pienso con mucho gusto $\mathrm{e}$ impaciencia en, al menos 2 meses de vacaciones" (II.12.), "vuelo a Madrid desde el aeropuerto de Berlin" (II.21.), "estas colas después del reparto" (III.7.), "la vida ya no se aparece a la de desde hace 20 años" (III.9.)

\section{CONCLUSIONES}

El aprendizaje de las preposiciones suele plantear problemas por su amplio número y por sus muchos valores. Así, para el aprendiz y el profesor se convierten en un obstáculo dificil de encarar. En principio parece que esta dificultad la corrobora nuestro estudio ya que el número de errores es elevado. Aún así, consideramos que una descripción detallada de los errores y los valores de las preposiciones muestran una delimitación de áreas en las que se debería hacer hincapié y que no suponen una tarea inabarcable.

Por otra parte, debemos recordar que el elevado número de enrores va paralelo a la elevada frecuencia de uso. En el corpus las preposiciones más utilizadas son tres ("de", "en" y "a"), entre las cuales representan el 70,031\%, cifra que alcanza el $88,853 \%$ si contabilizamos las tres preposiciones siguientes en frecuencia de uso ("con", "por" y "para"). Por lo tanto, parece lógico que el mayor número de errores se centre en estas preposiciones.

Según nuestro análisis los errores que más se generalizan son los siguientes:

$\mathrm{DE}$

- Régimen preposicional, principalmemte de verbos.

- Omisión de la preposición "de" precediendo a oraciones subordinadas.

- Omisión de la preposición "de" en locuciones preposicionales.

- Uso innecesario junto a otras preposiciones.

- Uso / elección en expresiones adverbiales.

EN

- Régimen preposicional, principalmente de verbos.

- Elección de "en" por "de" con valor de pertenencia o cualidad por interferencia del polaco.

- Uso / elección en expresiones adverbiales.

A

- Uso u omisión de la preposición delante de objetos directo.

- Régimen preposicional, principalmente de verbos. 
- Uso de "a" por "en" para expresar situación espacial.

- Uso de "a" por "de" para indicar cualidad, materia o tiempo.

\section{CON}

- Uso de "con" por "de" para indicar cualidad, naturaleza o condición de persona o cosa, por interferencia del polaco.

POR

- Uso de "por" por "para" con valor de finalidad.

\section{PARA}

- La idea de finalidad prevalece sobre otros valores.

Durante el proceso de aprendizaje se debería focalizar el mayor esfuerzo en estos casos, sobre todo en los primeros de las tres primeras preposiciones, y no dispersarlo por igual en la amplia variedad de usos y valores preposicionales. De esta forma el aprendizaje será mucho más efectivo, ya que nos centraremos en los puntos en los que realmente aparecen los problemas.

\section{BIBLIOGRAFÍA}

Fernández S. (1997), Interlengua y análisis de errores en el aprendizaje del español como lengua extranjera, Edelsa, Madrid.

Fernández Jódar R. (2004), El sistema verbal de la interlengua de los aprendices polacos de español, Studia Romanica Posnaniensia, XXXI, 311-319.

González Cremona P. y Palka E. (2004), Guía de pecadores, Księgarnia Akademicka, Cracovia.

González Hermoso A., Cuenot J.R. y Sánchez Alfaro M. (1994), Gramática de español lengua extranjera, Edelsa, Madrid.

Luque J.D. (1973), Las preposiciones. I. Valores generales. II. Usos idiomáticos, SGEL, Madrid.

Real Academia Española (1973), Esbozo de una nueva gramática de la lengua española, Espasa-Calpe, Madrid.

Sánchez A., Martín E. y Matilla J.A. (1980), Gramática práctica de español para extranjeros, SGEL. Madrid. 